\title{
Zur humanistischen Stadtgeschichtsschreibung in Böhmen, am Beispiel der Chronik der Stadt Kaaden Ein Beitrag zur historischen Fachsprachenforschung ${ }^{1}$
}

\author{
Lenka VODRÁŽKOVÁ
}

\begin{abstract}
On Humanistic urban history writing in Bohemia: A case study of the Kaaden town chronicle from the diachronic perspective as a contribution to historical research into professional language

The article presents a chronicle of the town of Kaaden (Kadaň) dating from the $16^{\text {th }}$ century, currently held in Prague's Monastery of Our Lady of the Snows. It explores several aspects of Humanistic urban history writing, including the presence of the author in the text of the chronicle, the methodology of the author's historiographic work, and his choice and use of language (German, Latin). The study also presents this chronicle as an interesting and important source of information on writing practices in north-west Bohemia from a text-analytical perspective.
\end{abstract}

Keywords: German, historiography, diachrony, Humanism, Bohemia

DOI: doi.org/10.15452/StudiaGermanistica.2020.26.0006

\section{Einleitung}

Was war diesem Buch eingeschrieben Worden, sol Niemandten zu Nahe sein. Dann es dahin gemeinett, das Man etzlicher Masen, den Ernst vndt Eifer auch die gefahr vndt schwere Muhe, derer Personen die sich gottes Worts hertzlich angenommen, Was sie Darbey erlitten, vnndt ausgestanden, Hören vnd Vernehmen. (fol. XVIIr).

Mit diesen Worten wird die Chronik der Stadt Kaaden/Kadaň eröffnet, ${ }^{2}$ die die Reihe der deutsch geschriebenen humanistischen Werke historiographischen Inhalts böhmischer bzw.

1 Der Begriff „Fachsprache“ wird im vorliegenden Beitrag in breiterem Rahmen als Gesamtheit der fundamentalen spezifischen sprachlichen Merkmale auf der Ebene der Textes, des Stils, der Syntax, der Lexik usw., die ein bestimmtes Fachgebiet oder einen bestimmten Sachbereich charakterisieren, aufgefasst. In Bezug auf diese Auffassung stellt der vorliegende Aufsatz eine Ausgangsbasis für die weitere ausführliche historiolinguistische Erforschung der Chronik der Stadt Kaaden dar, die auf die fachsprachlichen Spezifika dieses historiographischen Textes abzielt. Vgl. Reichmann (1996:119-133); Reichmann/Wegera (1988:IX-XIV); Seibicke (2003:2377-2391). Weiter vgl. dazu Eis (1971).

2 Mit dem vorliegenden Beitrag über die deutschgeschriebene Chronik von Kaaden, auf die im Jahr 2010 die Historikerin Marie Tošnerová (2010:111-115) in ihrer Monographie über die humanistische Stadtgeschichtsschreibung in Böhmen in den Jahren 1526-1620 hinwies, erweitert die Autorin ihre sprachhistorische Erforschung der humanistischen Chroniken der nordwestböhmischen Städte Komotau/Chomutov (2009, 2015) und Brüx/Most (2013, 2015). 
nordwestböhmischer Provenienz der zweiten Hälfte des 16. Jahrhunderts erweitert und die aus inhaltlicher und formaler Perspektive die Geschehnisse und gesellschaftlichen Verhältnisse sowie die Spezifika der schriftlichen Kultur der Zeit vor der Schlacht am Weißen Berg 1620 reflektiert.

\section{Zur humanistischen Stadtgeschichtsschreibung}

Die historiographischen Werke, ${ }^{3}$ die in der Zeit des Humanismus an Bedeutung gewannen, entstanden als Mittel zur Erfassung der neuen Lebensordnungen in Ländern und Städten mit dem Ziel, geschichtliche Begebenheiten bzw. Ereignisse politischer, religiöser und gesellschaftlicher Art in chronologischer Abfolge darzustellen. Im Zusammenhang mit der Rolle der Städte als wichtige Repräsentanten des kulturellen Geschehens stellte die Geschichte der (eigenen) Stadt („städtische Geschichtsschreibung ") einen der Hauptgegenstände der humanistischen Historiographie dar. ${ }^{4}$ Die Stadt und die Entfaltung ihrer Verwaltung, Kultur und Bildung mit neuen Bildungsmöglichkeiten in den Pfarr- und Stadtschulen bewirkten eine Umgestaltung der kommunikativen Verhältnisse und auch der Schriftkultur. Zu den Anfängen und zum allmählichen Aufschwung der städtischen Historiographie trugen vor allem der Ausbau der städtischen Administration, die Ausbildung eines spezifisch städtischen Selbstverständnisses und das Bedürfnis bei, diesem Selbstbewusstsein und dieser Emanzipation schriftlich Ausdruck zu verleihen (vgl. Schmidt 2000:1-17; Skála 1986:97-98). So umfasst die Stadtgeschichtsschreibung schriftliche Quellen, die in soziokulturelle Beziehungen und Atmosphäre innerhalb der Stadt und in die einzelnen Lebensbereiche ihrer Bewohner Einsicht nehmen lassen sowie gleichzeitig die Stellung des Autors - eines im Geiste des Humanismus erzogenen Bürgers - in der städtischen Gesellschaft und sein sprachliches Niveau dokumentieren.

\subsection{Humanistische Historiographie städtischer Provenienz in Böhmen}

In Böhmen erreichte die Stadtgeschichtsschreibung einen Höhepunkt in der zweiten Hälfte des 16. und am Anfang des 17. Jahrhunderts. ${ }^{5}$ Dies geht aus den günstigen Bedingungen für ihre Entstehung hervor - den relativ friedlichen, kriegslosen Lebensumständen, dem Humanismus und seiner Rezeption, der humanistischen Auffassung der Geschichte mit philologisch-historischen Bemühungen um den dokumentierenden Charakter der historiographischen Texte sowie der Ausbildung in einem relativ dichten Netz der städtischen Partikularschulen; die Lese-, Schreib- und Rechenfertigkeiten waren für die spätere Teilnahme der Bürger an der Verwaltung der Stadt unentbehrlich.

An der deutsch geschriebenen historiographischen Produktion in Böhmen in der sog. Vorweißbergischen Zeit, d. h. vor 1620, beteiligte sich wesentlich die nord- und nordwestböhmische Stadtgeschichtsschreibung, die ihre Spuren neben der Stadt Kaaden auch in Böhmisch Leipa/Česká Lípa, Brüx/Most, Görkau/Jirkov, Joachimsthal/Jáchymov, Komotau/Chomutov, Laun/Louny, Leitmeritz/ Litoměřice, und Teplitz/Teplice hinterließ; die Gründe liegen u. a. in den damaligen komplizierten konfessionellen Verhältnissen dieser Region.

Mit deutschgeschriebenen historiographischen Texten aus der Provenienz der böhmischen Länder beschäftigten sich aus historiolinguistischer Perspektive z. B. Libuše Spáčilová (,Chronik der Olmützer Bürgerfamilie Hobel (1530-1629)', 2005) oder Vlastimil Brom (,Der deutsche Dalimil. Untersuchungen zur gereimten deutschen Übersetzung der alttschechischen Dalimil-Chronik‘, 2006; ,Di tutsch kronik von Behem lant‘, 2009).

4 Der Hauptgegenstand der humanistischen Historiographie waren die Geschichte der eigenen Nation (,nationale Geschichtsschreibung“) oder die Geschichte der (eigenen) Stadt (,städtische Geschichtsschreibung“). Die Konzeption der im Sinne des mittelalterlichen Universalismus verfassten Weltgeschichte trat in der humanistischen Historiographie zurück. Vgl. Tošnerová (2010:21); Muhlack (2002:30-34).

Die städtische Historiographie in Böhmen hat sich im Vergleich mit den anderen Ländern später konstituiert, denn ,,bis zur hussitischen Revolution waren die Städte kein selbständiges politisches Subjekt“. Vgl. Bláhová (2000:233); Skála (1988:377-403). 


\section{Die Chronik der Stadt Kaaden}

Die Chronik der Stadt Kaaden und ihre Entstehung hängen mit der schwierigen Situation zusammen, in die sich die königliche Stadt Kaaden im 16. Jahrhundert wegen religiöser Streitigkeiten zwischen Katholiken und Protestanten begab: Der Konflikt spitzte sich dann mit der Verbreitung des Luthertums in der Region zu und es kam unter den Bewohnern der Stadt zu großen religiösen Auseinandersetzungen.

\subsection{Zur Beschreibung der Forschungsquelle}

Das Manuskript der ältesten Stadtchronik mit dem Titel ,Chronica Cadanensia' wird zurzeit in der Bibliothek des Franziskanerklosters zu Maria Schnee in der Prager Neustadt (Knihovna františkánů u P. Marie Sněžné na Novém Městě pražském) mit der Signatur Rf 13 aufbewahrt. ${ }^{6}$ Die Handschrift ist nicht vollständig überliefert; es fehlt das Titelblatt. Die Papierhandschrift hat insgesamt 219 Folios: Auf den nicht foliierten Index (fol. Ir - XVv) folgt das Vorwort (fol. XVIIr). Der foliierte Grundtext der Chronik fängt mit dem Jahr 821 an (fol. 1r), endet mit dem Jahr 1599 (fol. 167r) und zusätzlich wurden ihm Einträge aus den Jahren 1631-1659 (fol. 167v-170r) angehängt. Die letzten 32 Papierlagen der Chronik sind leer. Die Handschrift wird rechts oben mit schwarzer und roter Tinte foliiert; die mit arabischen Ziffern versehene Blätterbezeichnung beginnt im Grundtext mit Folio 1 auf dem Blatt 17r. Die Handschrift ist in einem festen hellbraunen Pappband gebunden, der wahrscheinlich aus dem 18 . Jh. stammt und $445 \times 320 \mathrm{~mm}$ groß ist. Auf dem Buchrücken befinden sich folgende Titel und Signaturen: Chronica Cadanensia (1. Zeile, schwarz), M. S. (2. Zeile, schwarz), in der Mitte ein großes schwarzes $R$ und unten eine schwarze 13. Die Blattgröße beträgt $310 \times 195 \mathrm{~mm}$. Der Text ist einspaltig und mit schwarzer Tinte geschrieben. Die Rubrizierung verdeutlicht als Gliederungs- und Ordnungselement, mit dem Überschriften, Jahr, Textanfang und Foliierung in der Chronik hervorgehoben werden, den Anfang und den Übergang der Kapitel. Die Schrift weist allgemeine Züge der neugotischen deutschen Kanzleikursivschrift des 16. Jahrhunderts eines erfahrenen und geschulten Schreibers auf.

\subsection{Zur Datierung der Chronik}

Obwohl das Manuskript der Chronik nicht datiert ist, lässt sich schlussfolgern, dass die Geschichte der Stadt Kaaden in den neunziger Jahren des 16. Jahrhunderts entstanden ist: Der Text reflektiert die zugespitzten Religionsverhältnisse zwischen Katholiken und Lutheranern in der Stadt und beschreibt die Ereignisse in Kaaden bis zur Erneuerung des Stadtrats im Jahr 1599. Vermutlich verfasste der Autor den Text der Chronik im Jahr 1597: Er arbeitete an seinem Werk intensiv, kontinuierlich und ununterbrochen vom Anfang bis zum Ende der Chronik. Während bis zum Jahr 1597 die Jahresangaben und die Foliierung sorgfältig mit roter Tinte geschrieben werden, wurden die Ereignisse in den letzten zwei Jahren 1598 und 1599 ohne verzierte Rubrizierung, wahrscheinlich zusätzlich nach der Beendung der Chronik, eingetragen.

\subsection{Zum Autor der Chronik}

Die handschriftliche Chronik der Stadt Kaaden stammt von einem unbekannten Schreiber; sein Name wird in der Chronik auch wegen des fehlenden Titelblatts nicht angeführt. Auf Grund der in der Chronik niedergeschriebenen Aufzeichnungen und ihres Inhalts lässt sich vermuten, dass der Schreiber aus der bürgerlichen Schicht kam, denn die soziale Stellung der Schreiber war eine weitere Voraussetzung für die Stadtgeschichtsschreibung. Aus dem Text der Chronik zum Jahr 1591 geht hervor, dass der Autor der Konfession nach ein Utraquist war:

Nach der Aufhebung des Franziskanerordens und Konfiszierung seines Besitztums befand sich die Klosterbibliothek in der Nationalbibliothek der Tschechischen Republik in Prag (Národni knihovna $\breve{C} R$ ). 
Jn diesem 91 Jar [...] sindt wir die sub utraque zum Cadan aus der Kloster Kirchen S. Michael genandt [...] aus beuehl Kays[erlicher] M[ayestät] [...] ausgetrieben worden. Welches gotteshaus wir derer sub utraque in die 44 Jar ruhlichen haben Jnne gehaltten. (fol. 160r)

Obwohl keine Informationen zur Ausbildung des Autors zur Verfügung stehen, griff hier wahrscheinlich kein beruflicher Schreiber zur Feder, sondern ein gut ausgebildeter Bürger, dessen Prestige mit dem in der Vorweißbergischen Zeit hochgeachteten Status eines Gelehrten innerhalb der Stadt stieg und der zu den Führungs- und Verwaltungseliten der Stadt gehörte.

Hinsichtlich der Beziehung des Autors zur Stadt lebte und schrieb der unbekannte Geschichtsschreiber der Kaadener Chronik - wie die weit überwiegende Mehrzahl der bürgerlichen Historiographen - nicht in Distanz zu seiner Stadt, sondern in unmittelbarer, unreflektierter Identifikation mit ihr; dies nahm einen zentralen Wert in ihrem Selbstbewusstsein ein. Dank der städtischen Politik und Selbstverwaltung war die wirtschaftliche und soziale Existenz der Autoren abgesichert und dank ihrer Nähe zum Rathaus und ihrem Anteil an der Stadtverwaltung genossen sie selbst und ihre Familien ein hohes gesellschaftliches Ansehen: Der Autor der Chronik von Kaaden kam aus dem Stadtverwaltungskreis, z. B. aus dem Ratsgremium, ${ }^{7}$ war vermutlich ein finanziell gut situierter Bürger, denn er konnte sich genug Zeit für das intensive historiographische Schaffen im Jahr 1597 nehmen, und hatte eine gute Übersicht über das Geschehen in der Vergangenheit und Gegenwart, was die Konzeption und der Inhalt der Chronik und ihre einzelnen Aufzeichnungen belegen.

\subsection{Die Chronik der Stadt Kaaden aus textologischer Perspektive}

Aus textologischer Sicht reflektiert die vorliegende Stadtchronik, die vorwiegend den Lebensbereich der Stadt Kaaden beschreibt, in Bezug auf ihre Kommunikations- und Textfunktion die Absicht des Emittenten, den Rezipienten über einen bestimmten Sachverhalt, bzw. bestimmte Ereignisse zu informieren, historische Fakten zu speichern und verfügbar zu machen (vgl. Brinker/Cölfen/ Pappert 2018:104-106, 136-138). Obwohl die Titelseite der Chronik aus unbekannten Gründen nicht überliefert ist, deutet das Manuskript mit seinem Textinhalt und -aufbau, seiner Ausstattung sowie der sorgfältigen Handschrift darauf hin, dass der Autor beabsichtigte, eine repräsentative historiographische Schrift zu verfassen. Er widmete der Vorbereitung der Arbeit außerordentliche Aufmerksamkeit, um so den Lesern historische Ereignisse von Kaaden möglichst klar zu vermitteln.

\subsubsection{Zum Textinhalt und -aufbau}

In Bezug auf den Textaufbau beginnt die Chronik der Stadt Kaaden wegen der Absenz der Titelseite mit einem Index bzw. einem Inhaltsregister, das eine alphabetische Liste von in der Chronik eingetragenen Ereignissen umfasst. Jedem Ereignis werden das jeweilige Jahr und der Hinweis auf das entsprechende Folio angeschlossen (nicht ursprünglich foliiert; fol. Ir-XVv). ${ }^{8}$ Wegen der fehlenden Foliierung lässt sich auch vermuten, dass der Index ursprünglich am Ende des ganzen Grundtextes der Chronik platziert wurde.

Den Anlass zur Entstehung der historiographischen Werke städtischer Provenienz, der nach dem damaligen Usus im Vorwort meistens angeführt wurde, gaben in der Regel das wachsende Selbstbewusstsein einiger Gruppen der Bürger in innerstädtischen Konflikten (1), die Streitigkeiten zwischen Städtern und Adel (2), die enge Beziehung zur Stadt (3), das Andenken an die eigene Person des Autors (4), der Nachlass für weitere Generationen der Stadtbevölkerung (5) und das Streben nach Anerkennung vom Stadtrat (6) (vgl. Tošnerová 2010:38-40). In der Chronik der Stadt Kaaden, an deren Entstehung die verschärften Religionsverhältnisse in der Stadt Anteil hatten, enthält das

7 Der Autor der Chronik von Kaaden war kein Stadtschreiber oder Stadtbeamter, der für Stadtbucheintragungen verantwortlich war. Bis heute wurde nämlich seine Schrift auf Grund des Vergleichs mit den Kaadener Stadtbucheintragungen aus der zweiten Hälfte des 16. Jahrhunderts nicht identifiziert.

8 Zur Foliierung der Kaadener Stadtchronik vgl. Tošnerová (2010:112, Anm. 4). 
kurze und bündige Vorwort (fol. XVIIr) die Absicht des Autors und deutet seinen Zugang zur Arbeit eines Chronisten sowie den Inhalt der Chronik an:

Dann es dahin gemeinett, das Man etzlicher Masen, den Ernst vndt Eifer auch die Gefahr vndt schwere Muhe derer Personen, die sich gottes Worts hertzlich angenommen, Was sie Darbey erlitten, vndt ausgestanden, Hören und Vernehmen [...] Demnach solches aber von dem gegentheil des glaubens aufgemercket herkömpt, Hilfft es besser zur Warheit, Dieselben Geschichte hab ich zu sammen geschrieben. (fol. XVIIr)

Im Vorwort schreibt der Autor, dass er bei seiner Arbeit von den Aufzeichnungen auf zerrissen hin vndt her geworffenen Schkarteken (fol. XVIIr) ausging. Deshalb weist er hier darauf hin, dass alle aufgezeichneten Ereignisse nicht wahrhaftig und historisch richtig beschrieben werden müssen: [So] wirdt wol geacht, Das es aller Dinge nicht so gar richtig sein Muss (fol. XVIIr). Gleichzeitig lässt er zusätzliche Korrekturen und Ergänzungen von Lesern der Chronik zu, aber er wehrt sich gegen eventuelle Einwände hinsichtlich der Ehrverletzung beteiligter Personen:

Nach der Jarzeitt kundt so daneben etwas anders denn die Religion betroffen, ist funden Worden, auch mit eingenommen, Vndt mir alleine (Niemandt zu wieder oder Nachtheil) Jnn ein Buch gehefftet, Wo einer solches lesen Möchte vnd die Acta richtieger Wuste, Stehett Jm frey eine Besserung zu Machen oder seine Wissentschafft fur sich selber zu behaltten. Es sol auch keiner Person, derer hierin gedacht Wirdt an Ehren verletzlich sein. (fol. XVIIr)

Das Vorwort endet mit einem Gebet, dessen Bestandteil ein Hinweis auf den Nachlass für weitere Generationen der Stadtbevölkerung ist: Gott erhaltte sein Wortt, die Edle Warheitt auf Vnsere Nachkomen. Amen (fol. XVIIr). Im Zusammenhang damit geht aus dem Vorwort hervor, dass der Autor mit der Veröffentlichung der Chronik, Wo einer solches lesen Möchte, rechnete (fol. XVIIr).

Dem Vorwort folgen Zitate in Form eines abgeschriebenen Auszugs aus der ,Kronika čzeska“ (Böhmische Chronik, 1541) Václav Hájeks von Libotschan (Václav Hájek z Libočan, †1553), der die Anzahl der Städte, Dörfer, Schlösser und Besitztümer beschreibt (fol. XVIIv). Der Autor ging hier vermutlich von der deutschen Übersetzung der ,Böhmischen Chronik` Václav Hájeks von Libotschan aus, die 1596 von dem Kaadener Stadtnotar Johann Sandel $(\dagger 1625)^{9}$ aus dem Tschechischen ins Deutsche übersetzt wurde. Der Autor der Chronik von Kaaden stützt sich bei seiner historiographischen Arbeit auf Werke, die zum wesentlichen Teil in der zweiten Hälfte des 16. Jahrhunderts, also zu Lebzeiten des Autors der Chronik, in der Druckversion verbreitet wurden und ihm gut bekannt waren.

Der eigentliche Text der Chronik beginnt mit dem Jahr 821, als laut Václav Hájek von Libotschan die Stadt Kaaden gegründet wurde. Die Aufzeichnungen enden im Jahr 1599 mit der Ernennung des neuen Stadtrats in Kaaden. Die einzelnen inhaltlichen Abschnitte beginnen mit der historischen Datierung nach Jahren (fol. 1r-167r), eventuell auch Tagen (z. B. 1575: Sonnabentt den 3. Septembris, [fol. 146v], Mittwoch den 13. Septembris [fol. 147v]) oder auch mit einer, meistens rubrizierten Überschrift (z. B. Eine andere Christliche tagweise [fol. 17r], Jm Thon, O Reicher Bott im Trone [fol. 18v]) bzw. mit der mit Rubrizierung verzierten ersten Zeile (z. B. Jnn diesem Jahr ist der Landtag gehaltten [fol. 131r]). Die Eintragungen sind meistens chronologisch geordnet. Die Konzeption des eigentlichen Textes der Chronik korrespondiert mit dem Usus der historiographischen Werke: Seit dem 15. Jahrhundert erscheinen in den Stadtbüchern der böhmischen Städte Einträge, die bedeutenden lokalen Ereignissen gewidmet sind (vgl. Tošnerová 2010:11). So wurden in der Chronik von Kaaden neben den Aufzeichnungen über wichtige historische Ereignisse in der Stadt auch Informationen über das Geschehen in anderen Städten in der nahen Umgebung (z. B. Brüx [fol. 14v], Joachimsthal [15r]) niedergeschrieben.

Neben den historischen Ereignissen reflektiert die Chronik die komplizierten Religionsverhältnisse zu Ende des 16. Jahrhunderts sowohl im Land, als auch in der Stadt, denen der unbekannte

In Kaaden war Johann Sandel in den Jahren 1594-1601 tätig. 
Autor eine besondere Aufmerksamkeit widmete. Das Gebiet Nordwestböhmens, wo der Autor lebte und wirkte, war protestantisch geprägt und stand somit in enger Verbindung mit Sachsen: Die nordwestböhmische Region bildete im 16. Jahrhundert eine wichtige Brücke zwischen Böhmen und Sachsen, über die frühe lutherische Reformgedanken strömten. Durch das ganze Jahrhundert und noch bis zum Jahr 1624 dauerte der Zug protestantischer Geistlicher aus Sachsen nach Böhmen an. ${ }^{10}$ Die Geschichte der Stadt Kaaden wird meist chronologisch nach den Jahren geschildert und jeder Eintrag stellt eine inhaltliche Einheit in einem Absatz der Chronik dar. Einige Jahre werden vermutlich wegen mangelnder Informationen, die zum Eintrag geeignet waren, ausgelassen. Umfang und Form der einzelnen Aufzeichnungen sind unterschiedlich: Einige Einträge enthalten kurze, einzeilige Informationen, andere kontinuierliche Erzählungen. In der Chronik behandelt der Autor, der sich wahrscheinlich auch stark in konfessionellen Angelegenheiten engagierte, ausführlich die Hussitenkriege im 15. Jahrhundert und auch das Jahr 1575, als über die konfessionellen Verhältnisse in den böhmischen Ländern auf der Ebene des Landtags verhandelt wurde. Der umfangreichste Eintrag, der 13 Folioblätter beträgt, bezieht sich auf das Jahr 1534, als zum ersten Mal die Namen der Kaadener Stadtratsmitglieder angeführt werden (fol. 78v-90r). Die Chronik informiert ausführlich über die Verhandlungen in Kaaden, die mit einem Vertrag zwischen den Habsburgern und den deutschen Protestanten endeten. Über die Ereignisse seit den siebziger Jahren des 16. Jahrhunderts schrieb der Autor wahrscheinlich aus eigener Erfahrung, was die zunehmende Anzahl von Einträgen zu den einzelnen Jahren sowie die Hervorhebung von Alltagsereignissen belegen.

Zwischen die einzelnen Einträge sind Abschriften der Dokumente, die sich vor allem auf die Religionsproblematik beziehen, Abschriften der Lieder und verschiedene kurze Geschichten eingegliedert (z. B. fol. 16v, 19r-19v, 40v, 51v).

Die eigentlichen chronikalischen Aufzeichnungen begleiten die Auflistungen als fester Bestandteil der Texte dokumentierenden Charakters (z. B. fol. 13r). Mit dem Verzeichnis der neuen Stadtratsmitglieder im Jahr 1599 wird die Chronik abgeschlossen (fol. 167r).

Die Chronik enthält nicht nur zahlreiche Informationen über die Geschichte der Stadt, sondern auch über das Leben in der Stadt in der Vorweißbergischen Zeit. Obwohl ihr Schwerpunkt in der Schilderung der Ereignisse im 16. Jahrhundert liegt, stellt die Chronik der Stadt Kaaden mit ihrem inhaltlichen Umfang der Eintragungen von historischen Ereignissen von 821-1599 eine außerordentliche Quelle historiographischen Charakters dar. Ihre Konzeption und der Stil der Einträge belegen, dass ihr Autor nicht nur gut ausgebildet war, sondern dass er eine Übersicht über das vergangene und zeitgenössische Geschehen in der Stadt und im Land sowie über andere historiographische Literatur hatte.

\subsubsection{Zur historiographischen Methodologie}

Hinsichtlich der methodologischen Bearbeitung der historischen Ereignisse trägt der Text wichtige Merkmale des humanistischen Schrifttums: Der Text enthält zahlreiche Abschriften der Dokumente (z. B. von Urkunden, Stadtbucheintragungen sowie städtischen Aktenstücken, in denen verschiedene historische Ereignisse mit Beziehung zur Geschichte von Kaaden beschrieben werden), die vor allem die Geschichte der Stadt und die Religionsverhältnisse betreffen. Im Text befinden sich auch die bibliographischen Hinweise auf die Autoren, Werke, denen aber nur selten die Seitenbzw. Folienangaben, wo sich der jeweilige Textausschnitt befindet, eventuell auch die Namen der einzelnen Kapitel des zitierten oder recherchierten Werkes angehängt werden (z. B. fol. 63r). Die in chronologischer Abfolge der zusammengestellten Informationen bieten dann dem Leser eine Übersicht über Ereignisse in der Stadt Kaaden oder im Land, die im Zusammenhang mit Kaaden stehen (z. B. die erste historische Erwähnung der Stadt im Jahr 821; die Berufung der Stadtratsmitglieder zur Böhmischen Kammer nach Prag wegen freiwilliger Ernennung des Dekans und der Gründung

10 Das böhmisch-sächsische Erzgebirge stellte ein einzigartiges Gebiet dar, das mit einem dichten Netz der grenzüberschreitenden kulturellen, religiösen, politischen und wirtschaftlichen Bindungen durchwebt wurde: Es kamen Ansiedler aus Sachsen hierher, die auf den Grundherrschaften des böhmischen Adels ein Betätigungsfeld fanden. Von Bedeutung war der Wechselverkehr im Bereich des Hochschulwesens, vor allem an den Universitäten in Leipzig und Wittenberg. 
der Schule, wofür die Repräsentanten der Stadt bestraft wurden). Diese methodologische Verfahrensweise dokumentiert die Arbeit eines gut ausgebildeten Humanisten, der sich mit den Methoden der Geschichtsschreibung wahrscheinlich während seines Universitätsstudiums bekannt gemacht hatte. Mit seiner Konzeption und Auffassung des behandelten Stoffes stellt das Manuskript eine bemerkenswerte Quelle zur Erforschung der Methodologie der humanistischen Arbeit auf dem Gebiet der Historiographie dar.

\subsubsection{Zum sprachlichen Aspekt}

Nach dem Regierungsantritt und der Machtübernahme der Habsburger (1526) verbreitete sich das Deutsche in den böhmischen Ländern neben dem Tschechischen als zweite Landessprache. Die von der deutschen Bevölkerung besiedelten Grenzgebiete waren vorwiegend deutschsprachig. ${ }^{11}$ Das Tschechische war im 15. Jahrhundert als (städtische) Amtssprache üblich und auch in der Vorweißbergischen Zeit wurde in den meisten Städten, vor allem im Inland der böhmischen Länder, Tschechisch gesprochen. Ein umfassend gebildeter Mensch sollte - mit Änderung der Bewertung der Kenntnis fremder Sprachen - außer Volkssprachen ${ }^{12}$ auch Latein (und ein wenig Griechisch) beherrschen (vgl. Glück/Klatte/Spáčil/Spáčilová 2002:VIII-IX). So entfaltete sich der Humanismus in den böhmischen Ländern bis ins 17. Jahrhundert in drei Sprachen: Latein, Deutsch und Tschechisch.

Obwohl in den Grenzgebieten der böhmischen Länder die deutsche Sprache vorherrschend war (z. B. war Kaaden im 16. und 17. Jahrhundert eine überwiegend deutschsprachige Stadt), ${ }^{13}$ gab es auch hier Bürger, die beide Sprachen - Deutsch und Tschechisch - beherrschten (z. B. gab es ab 1470 in Kaaden neben dem deutschen auch einen tschechischer Stadtschreiber). Auch die historiographischen Werke wurden in dieser Region in beiden Volkssprachen verfasst; Deutsch und Tschechisch sind hier gleichermaßen vertreten; auf Latein wurden Abschriften, Verse und Chronogramme geschrieben (vgl. Tošnerová 2010:34-35).

Die Chronik der Stadt Kaaden ist auf Deutsch und Latein geschrieben. Der Grundtext ihres Manuskriptes ist mit Rücksicht auf das Streben nach Objektivität bei humanistischen Verfassern von historiographischen Werken und im Zusammenhang damit aus Rücksicht auf die exzerpierten Quellen in der unpersönlich erzählenden Perspektive der 3. Person Singular (Er-Form) geschrieben. Die Chronik belegt aber auch den persönlichen Anteil ihres Autors an historischen Geschehnissen der Stadt. Die Rolle des Autors als Augenzeugen projiziert sich in die Form der 1. Person Plural (wir), mit der sich der Autor der Chronik mit den Ereignissen identifiziert. Das Personalpronomen wir kommt im Eintrag zum Jahr 1591 vor, wo der Autor zu den Utraquisten Stellung nimmt:

In diesem 91 Jar [...] sindt wir [fett; L. V.] die sub utraque zum Cadan aus der Kloster Kirchen S. Michael genandt [...] aus beuehl Kays[erlicher] M[ayestät] [...] ausgetrieben worden. Welches gotteshaus wir [fett; L. V.] derer sub utraque in die 44 Jar ruhlichen haben Jnne gehaltten. (fol. 160r)

Im Eintrag zum Jahr 1592 nahm der Autor an der Reise der Repräsentanten der Stadt Kaaden nach Prag teil, was die im Text verwendete 1. Person Plural belegt:

Inn diesem 92 Jar den 6. Octobris, welches war Montag, Sindt Fünff Personen von Caden als D. Simon Florin, Adamus Beyer, Cyprianus Stierba, Zacharias Schönhöfer und Thomas Zikan (Veit Thim aber hatt auch mit gen Praga reisen sollen, aber wegen leibes schwachheit Verschönet worden), in

11 Bis zum zweiten Jahrzehnt des 15. Jahrhunderts dominierten ein deutscher Rat und eine deutsche Verwaltung die meisten Städte, denn die deutschen Kolonisten brachten das deutsche Stadtrecht ins Land und gaben den jungen Städten Verfassungen nach diesem Recht. In den Stadtkanzleien waren bereits seit dem 14. Jahrhundert neben dem Lateinischen auch Deutsch und Tschechisch benutzt worden.

12 Der Begriff „Volkssprache“ wird im vorliegenden Beitrag im Sinne der eigenen - gesprochenen und geschriebenen Sprache der jeweiligen Sprachgemeinschaft als Gegensatz zur lateinischen Bildungssprache verwendet.

13 Im 13. Jahrhundert erfolgte hier ein Zuzug von sächsischer und fränkischer Seite. Mit der Vermehrung der religiösen Konflikte bewegten sich die zahlreichen Bewohner zur Abwanderung nach Sachsen und der Anteil der tschechischen Bewohner nahm im Gegenzug zu. Hierzu vgl. Skála (1968:12). 
die bömische Cammer gefodert Vnd eingelassen worden, haben alda 17 Herren Herrn Standtes gesessen, Der Landhoffmeister Lochwitzer hat wortt geredt. Ist vns [fett; L. V.]. Vorgehalten worden, das wir frembte auslendische Vnordentliche Prister die die Consistorio zu Prag nicht zu gethan, haltten Vnd födern Vnd nun Viel Jar lang dieselben geehret vnd genehret haben. Auch das wir [fett; L. V.] ohne erlaubnus Kay[serlicher] M[ayestät] eine Kirchen erbauet haben, Neben auch eine sonderliche Schuel vnsers gefallens haltten Vnd anrichten, vnd noch Viel ander artickel mehr, Welche nicht alle zu erzelen. Derweg[en] wir [fett; L. V.] in Weysen thurm zu gehen Verurteilet worden [...] (fol. 161r)

Es ist dabei zu beachten, dass der darauf folgende Text ursprünglich in der Form der 3. Person Plural (sie) geschrieben, aber das Personalpronomen sie später gestrichen wurde und mit gleicher Hand zur Form der 1. Person Plural (wir) korrigiert wurde.

Die eigene Erfahrung zeigt sich in der Chronik in der Schilderung des Alltagslebens in der Stadt, die ab den Einträgen zu den siebziger Jahren des 16. Jahrhunderts wesentlich zunehmen, und sie wird noch durch die 1. Person Singular (Ich-Form) verstärkt, z. B. im Eintrag, der sich auf die Teuerung im Jahr 1571 bezieht: „wie ich denn selbst geschrieben und gesehen“ (fol. 122v).

Der Autor bemühte sich um eine objektive Darstellung der historischen Ereignisse, aber trotzdem ist aus dem Text ab und zu seine starke Bindung an die evangelische Kirche ersichtlich.

Die deutsch geschriebenen Textabschnitte der Chronik der Stadt Kaaden sind enthalten:

- im Grundtext der Chronik,

- in Marginalien zum Inhalt, die z. B. Eigennamen (Zischka [fol. 6v]), Albrecht Schlick [fol. 74r], Phillipus Melanthon [fol. 8v]), Orte (Kloster zu Wittenberg [fol. 4r], Constandinopel [fol. 7r]), Stichwörter (Brand [fol. 5r], Schnee [fol. 8r], Türkenzug [fol. 98r], [fol. 106r]), Ereignisse (Buchtruck erfunden [fol. 7r], Brandt zum Cadan [fol. 8r], Orgeln gesetzet [fol. 12r], Befehl wegen der Schul [fol. 76r], Martinus Luther stirbt [fol. 108v], Kirchstag zu Speyer 1570 [fol. 122r]) betreffen, und zu exzerpierten Quellen (z. B. zur Bibel, S. Joannes [fol. 3v]); die einzelnen Stichwörter dienen neben den roten Unterstreichungen (z. B. von Aufzählungen (z. B. Zum ersten, Zum andern, Zum dritten usw. [fol. 44r - 45r]), Datierungen (z. B. Den 18. Nouembris [fol. 155v]) zur besseren Orientierung im Text;

- in Zitaten (z. B. in Zitaten aus einem 1538 aufgeführten Fastnachtspiel [fol. 99r-99v]), Abschriften der Dokumente und der Lieder, die vor allem die Religionsverhältnisse im Land und in der Stadt betreffen (z. B. die Abschriefft der ersten eingeleibtenn Vereinigung Inn die Landtafel, glaubens Wegen zu beiden Teilen (fol. 44r); mehrere abgeschriebene Texte beziehen sich auf das Jahr 1530 (fol. 46r-69r), z. B. Einen Klag der Papisten wieder die Euangelischen (fol. 46v-48r), Entschuldigung der Euangelischen wieder die Vngegrundte Vnwarhaffte klage [...] (fol. 48v-51v), Schriefft an den Wolgebornen Herren Herren Lorentz Schlicken (fol. 51v-52v) usw.;

- in Auflistungen (z. B. in statistischen Angaben zu Städten, Dörfern usw. [fol. 13r], Übersichten über die Finanzierung der Pfarreien [fol. 24v-25v], Informationen über Personen und Pferde in Kriegszügen [fol. 41v-43r]), Verzeichnissen der Bewohner von Kaaden 1574, die sich um das ursprüngliche Minoriten-Kloster kümmerten [fol. 129v130v], Verzeichnissen der Stadtratsmitglieder von den Jahren 1530 [fol. 46r], 1536 [fol. 97v], 1538 [fol. 99v-100r], 1539 [fol. 103r] usw.

Neben dem Deutschen bedient sich der Autor der Chronik auch des Lateinischen; diese Tatsache deutet auf seinen Bildungsstand hin. Das Lateinische übte bis gegen Ende des 18. Jahrhunderts wichtige Funktionen aus. Es war nicht nur die Sprache der antiken Kultur und der christlichen Welt, namentlich der katholischen Kirche, sondern auch die Sprache des Humanismus, der Literatur und der höheren Bildung. Auf Latein geschriebene Textabschnitte der Chronik der Stadt Kaaden sind enthalten:

- im Titel der Chronik (Chronica Cadanensia); es handelt sich aber um einen zusätzlichen Titel aus dem 18. Jahrhundert; 
- in Zitaten und Abschriften der lateinischen Werke und Lieder (z. B. Carmen in Monasterion quodam, fol. 20r);

- in Marginalien, z. B. in Eigennamen (Carolus Quinto [fol. 18v, Magister Simon

[fol. 28v], Maxmilianus [fol. 111v]), Stichwörtern (Historia [fol. 150v]).

In sprachlicher Hinsicht ist zu bemerken, dass das Manuskript in der späteren Etappe des Frühneuhochdeutschen entstanden ist, in der ,überregionale Erscheinungen zu wichtigen Charakteristika der Sprache gehören, während regionale sprachliche Merkmale allmählich überwunden wurden" (vgl. Spáčilová 2005:36). Im Text zeigen sich die Vereinheitlichungstendenzen im Frühneuhochdeutschen, die allmählich zur Herausbildung der neuhochdeutschen Schriftsprache führten (z. B. wenige graphische Varianten und Reduktion der sprachlichen Formenvielfalt des Deutschen in Texten aus dem 16. und 17. Jahrhundert) (vgl. Knape 2000:1676).

\section{Fazit}

Historiographische Werke städtischer Provenienz des 16. und beginnenden des 17. Jahrhunderts tragen als wichtige schriftliche Quellen aus inhaltlicher und formaler bzw. sprachlicher Sicht wesentlich zur Erkenntnis der individuellen und kollektiven Identität des Bürgertums sowie zur Beleuchtung seines Weltbildes und Lebensgefühls in der Vorweißbergischen Zeit am Ende des 16. und am Anfang des 17. Jahrhunderts bei. Die Geschichte der Stadt Kaaden, die von der humanistischen Schreibpraxis eines Repräsentanten der bürgerlichen Schicht Zeugnis gibt, bereichert in dieser Hinsicht vor allem die Erfahrungen über das Niveau der Schreibtätigkeit und über die methodologische Herangehensweise der historiographischen Bearbeitung der Stadtgeschichte im Kontext der Sprach- und Religionsverhältnisse sowie der historiographischen Fachprosa in einem der Randgebiete Böhmens am Ende des 16. Jahrhunderts, wo einer der deutschsprachigen Chronisten den Wunsch hatte, mit seinem Werk „die Edle Warheitt auf Vnsere Nachkomen“ (fol. XVIIr) zu erhalten.

\section{Literaturverzeichnis}

\section{Primärliteratur:}

Chronica Cadanensia. Knihovna františkánů u P. Marie Sněžné na Novém Městě pražském [Bibliothek des Franziskanerklosters zu Maria Schnee in der Prager Neustadt], Sign. Rf 13.

\section{Sekundärliteratur}

BLÁHová, Marie (2000): Stadt, Bürgertum und Städtewesen im Spiegel der Geschichtsschreibung. Wege zur städtischen Historiographie in den böhmischen Ländern im Spätmittelalter und in der frühen Neuzeit. In: JoHAneK, Peter (Hrsg.): Städtische Geschichtsschreibung im Spätmittelalter und in der frühen Neuzeit. Köln; Weimar; Wien, S. 233-246.

Brinker, Klaus / Cölfen, Hermann / PAPPERT, Steffen (2018): Linguistische Textanalyse. Eine Einführung in Grundbegriffe und Methoden. 9., durchgesehene Aufl. Berlin.

EIs, Gerhard (1971): Forschungen zur Fachprosa. Ausgewählte Beiträge. Bern; München.

GlÜCK, Helmut / KLATTE, Holger / SpáčIL, Vladimír / SpáčILová, Libuše (2002): Deutsche Sprachbücher in Böhmen und Mähren vom 15. Jahrhundert bis 1918. Eine teilkommentierte Bibliographie. Berlin; New York.

KnAPE, Joachim (2000): Das Deutsch der Humanisten. In: Besch, Werner / Betten, Anne / ReICHManN, Oskar / SonderegGer, Stefan (Hrsg.): Sprachgeschichte. Ein Handbuch zur Geschichte der deutschen Sprache und ihrer Erforschung. 2., vollständig neu bearb. und erw. Auflage. 2. Teilbd. Berlin; New York, S. 1673-1681 [Handbücher zur Sprach- und Kommunikationswissenschaft, hrsg. v. Armin Burkhardt, Hugo Steger und Herbert Ernst Wiegand, Bd. 2.2].

MuHLACK, Ulrich (2002): Humanistische Historiographie. In: Helmrath, Johannes / MuHLACK, Ulrich / Walther, Gerrit (Hrsg.): Diffusion des Humanismus. Studien zur nationalen Geschichtsschreibung europäischer Humanisten. Göttingen, S. 30-34. 
Reichmann,Oskar/Wegera,Klaus-Peter(Hrsg.)(1988):FrühneuhochdeutschesLesebuch.Tübingen.

Reichmann, Oskar (1996): Autorenintention und Textsorte. In: Grosse, Rudolf / Wellmann, Hans (Hrsg.): Textarten im Sprachwandel - nach der Erfindung des Buchdrucks. Heidelberg, S. 119-133.

Rukovět humanistického básnictví v Čechách a na Moravě [Handbuch der humanistischen Dichtung in Böhmen und Mähren]. Bearb. v. TruhláŘ, Antonín / Hrdina, Karel / HeJnIC, Josef und MartíNEK. Jan.Bd. 2 und 3. Praha 1966, 1969.

SchмIDT, Heinrich (2000): Bürgerliches Selbstverständnis und städtische Geschichtsschreibung im deutschen Spätmittelalter. Eine Erinnerung. In: JoHANEK, Peter (Hrsg.): Städtische Geschichtsschreibung im Spätmittelalter und in der frühen Neuzeit. Köln; Weimar; Wien, S. 1-17.

SEIBICKE, Wilfried (2003): Fachsprachen in historischer Entwicklung. In: BESCH, Werner / BETTEN, Anne / Reichmann, Oskar / SonderegGer, Stefan (Hrsg.): Sprachgeschichte. Ein Handbuch zur Geschichte der deutschen Sprache und ihrer Erforschung. 2., vollständig neu bearb. und erw. Auflage. 3. Teilbd. Berlin; New York, S. 2377-2391 (Handbücher zur Sprach- und Kommunikationswissenschaft, hrsg. v. Armin Burkhardt, Hugo Steger und Herbert Ernst Wiegand, Bd. 2.3).

SKÁla, Emil (1968): Die Entwicklung der Sprachgrenze in Böhmen von 1300 bis etwa 1650. In: Acta Universitatis Carolinae - Philologica 5, Germanistica Pragensia V, Praha, S. 7-16.

SKálA, Emil (1986): Humanismus in den böhmischen Ländern als neue Bildungsbewegung. In: Philologica Pragensia - Časopis pro moderní filologii 68, Nr. 2, Praha, S. 97-98.

SKála, Emil (1988): Deutsche und tschechische Fachprosa in Böhmen in der Epoche des Humanismus. In: Harder, Hans-Bernd / Rothe, Hans / KoláR, Jaroslav / Wollman, Slavomír (Hrsg.): Studien zum Humanismus in den böhmischen Ländern. Köln; Wien, S. 377-403 (Schriften des Komitees der Bundesrepublik Deutschland zur Förderung der Slawischen Studien, hrsg. v. Hans Rothe, Bd. 11).

SpÁčILOvá, Libuše (2005): Chronik der Olmützer Bürgerfamilie Hobel (1530-1629). Ein Beitrag zur Geschichte des Frühneuhochdeutschen in Olmütz. Olomouc (Olmützer Schriften zur deutschen Sprachgeschichte, hrsg. v. Libuše SpéčIloví, Bd. 1).

ToŠNERová, Marie (2010): Kroniky českých měst z predbělohorského období. Úvod do studia městského kronikářství v Čechách v letech 1526-1620 [Die Chroniken der böhmischen Städte aus der Vorweißbergischen Zeit. Eine Einleitung in das Studium der Stadtgeschichtsschreibung in Böhmen in den Jahren 1526-1620]. Praha [Studie o rukopisech MONOGRAPHIA, Bd. XV.].

VoDrÁžKovÁ, Lenka (2009): Matthaeus Meisner und seine Chronik von Komotau. Ein Beitrag zur deutschsprachigen Geschichtsliteratur in Nordböhmen in der Zeit des Humanismus. In: MosHöVEL, Andrea / SpáčIlová, Libuše (Hrsg.): Kanzleisprache - ein mehrdimensionales Phänomen. Tagungsband für Prof. PhDr. Zdeněk Masařík, DrSc., zum 80. Geburtstag. Wien, S. 225-242 [Beiträge zur Kanzleisprachenforschung, hrsg. v. Jörg MeIER und Arne ZIEGLER, Bd. 6].

VODRÁŽKOVÁ, Lenka (2013): Zu historiographischen Texten in den böhmischen Ländern im 16. Jahrhundert, am Beispiel der Geschichte der Stadt Brüx des Humanisten Matthaeus Meisner. In: Ference, Anja Edith / SpéčILoví, Libuše: Deutsch als Sprache der (Geistes)Wissenschaften. Linguistik. Brno, S. 125-142.

VoDRÁŽKová, Lenka (2015): Sprachen und die humanistische Schreibpraxis in Böhmen: eine Fallstudie. In: Kusová, Jana / Malechová, Magdalena / VodrážKová, Lenka (Hrsg.): Deutsch ohne Grenzen. Linguistik. Brno, S. 81-101.

WINTER, Zikmund (1890, 1892): Kulturni obraz českých měst. Život veřejný v XV. a XVI. věku [Ein kulturelles Abbild der böhmischen Städte. Das öffentliche Leben im XV. und XVI. Jahrhundert]. 2 Bde. o. O.

Wriedt, Klaus (2000): Bürgerliche Geschichtsschreibung im 15. und 16. Jahrhundert. Ansätze und Formen. In: JoHANEK, Peter (Hrsg.): Städtische Geschichtsschreibung im Spätmittelalter und in der frühen Neuzeit. Köln; Weimar; Wien, S. 19-50. 


\section{Bilderanlagen:}

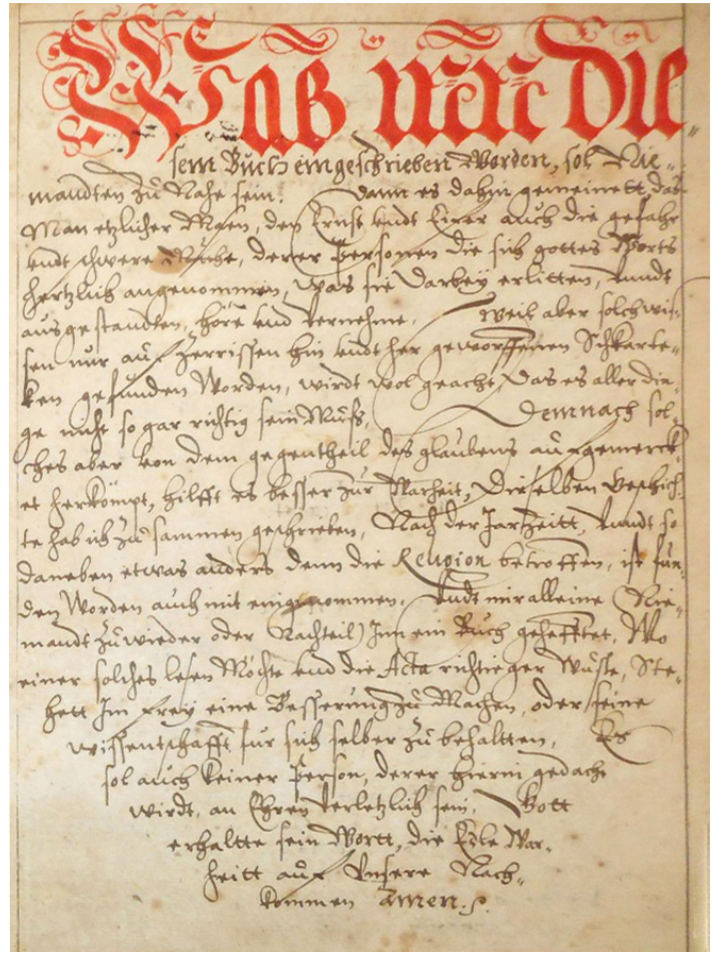

Abb.1: Chronica Cadanensia. Knihovna františkánů u P. Marie Sněžné na Novém Městě pražském [Bibliothek des Franziskanerklosters zu Maria Schnee in der Prager Neustadt], Sign. Rf 13, fol. XVIIr (Vorwort).

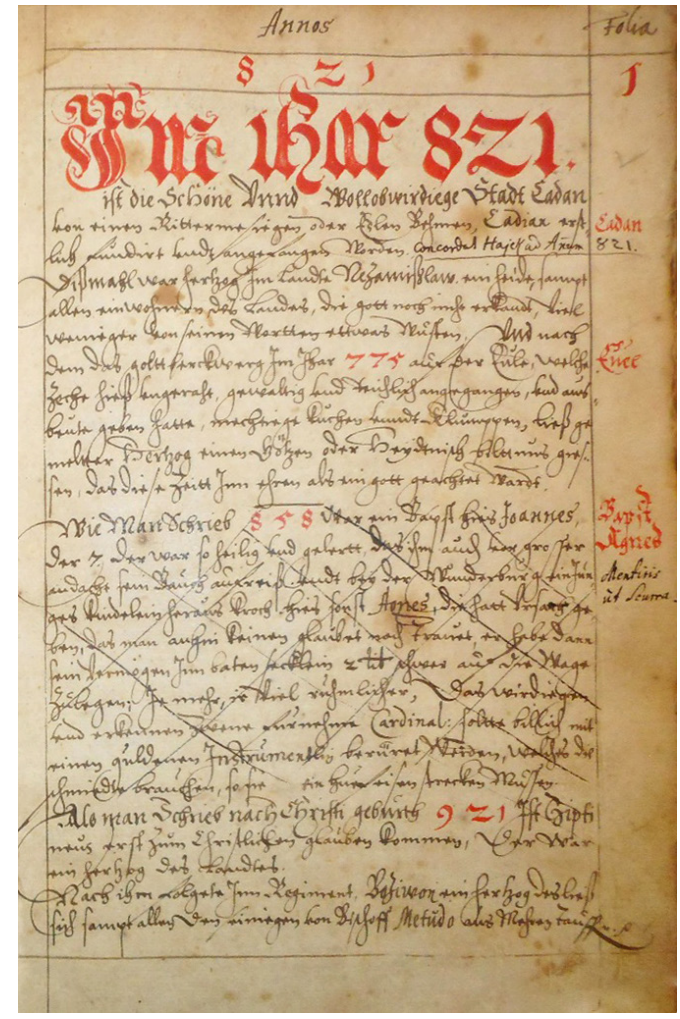

Abb.2: Chronica Cadanensia. Knihovna františkánů u P. Marie Sněžné na Novém Městě pražském [Bibliothek des Franziskanerklosters zu Maria Schnee in der Prager Neustadt], Sign. Rf 13, fol. 1r (Erste chronikalische Aufzeichnung über die Gründung der Stadt im Jahr 821). 


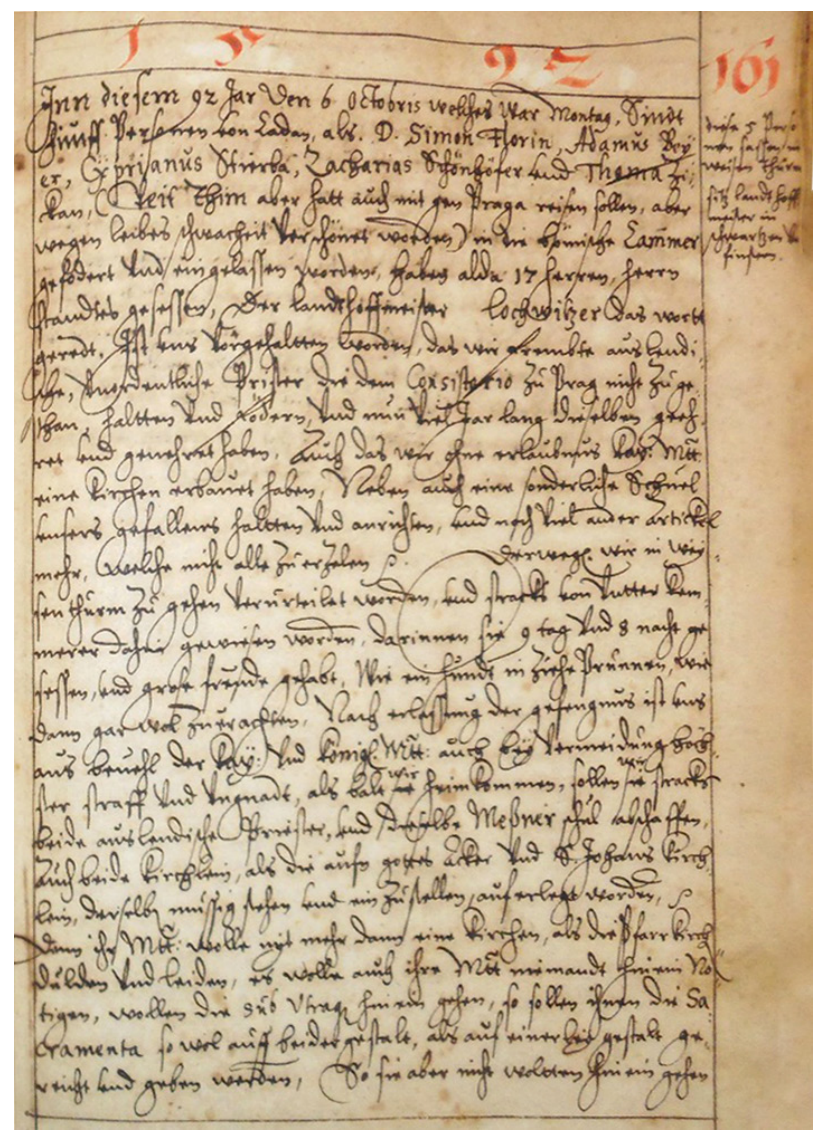

Abb. 3: Chronica Cadanensia. Knihovna františkánů u P. Marie Sněžné na Novém Městě pražském [Bibliothek des Franziskanerklosters zu Maria Schnee in der Prager Neustadt], Sign. Rf 13, fol. 161r (Chronikalische Aufzeichnung über die Reise der Repräsentanten der Stadt Kaaden nach Prag im Jahr 1592, an der der Autor beteiligt war [1. Person Plural wir]). 